\title{
A Model Describing the Relationship between Hot Rolling Ratio and Charpy Shelf Energy*
}

\author{
By Hiroyoshi MATSUBARA, ,* Tatsumi OSUKA, ${ }^{* *}$ Isao KOZASU, ,** and Koshiro TSUKADA**
}

The effect of hot rolling on ductility can be understood in terms of the improvement of as-cast structure by forging effect and change of shape and directionality of elongated non-metallic inclusions. In industrial rolling of hot strips and plates where rolling ratio is more than 20 to 30 , the latter factor predominates. In this note, an attempt to describe the change of Charpy shelf energy with cross rolling ratio will be made assuming a simplified model, and subsequently a normalized shelf energy independent of cross rolling ratio will be given.

The information on the relation between cross rolling ratio and shelf energy so far reported may be briefly summarized as below. In the following, $L$ is the final rolling direction and $T$ denotes transverse direction to $L ; R$ is the total rolling ratio (initial thickness/final thickness), and $R_{L}$ and $R_{T}$ are the rolling ratio in $L$ and $T$ directions respectively; and ${ }_{v} E_{s L}$ and ${ }_{n} E_{s T}$ are the shelf energy in $L$ and $T$ direction.

(i) As $R_{L}$ is increased, ${ }_{n} E_{s T}$ is decreased. ${ }^{1,2)}$

(ii) ${ }_{n} E_{s L} /{ }_{v} E_{s T}$ is a function of $R_{L} / R_{T}$, and for values of $R_{L} / R_{T}$ higher than 30 , it approaches to a value of about 3.0 to $3.5 .^{3,4)}$ One directional rolling such as hot strip mill rolling results in ${ }_{v} E_{s L} /{ }_{v} E_{s T}=3.5$.

(iii) The above two relationships are symmetrical in $L$ and $T$ directions.

These three relations indicate that ${ }_{v} E_{s L}$ and ${ }_{v} E_{s T}$ can be expressed as a decreasing function of $R_{T}$ and $R_{L}$ respectively, for example in terms of $1 / R_{T}$ or $-\log R_{T}$. In the present case, taking $1 / R_{T}$ for simplicity, one obtains (1) and (2) from the relation (i),

$$
\begin{aligned}
& { }_{n} E_{s L}={ }_{v} E_{s}^{*}\left(1+\alpha / R_{T}\right) \ldots \\
& { }_{v} E_{s T}={ }_{n} E_{s}^{*}\left(1+\alpha^{\prime} / R_{L}\right) .
\end{aligned}
$$

where $\alpha$ and $\alpha^{\prime}$ are constants, and ${ }_{v} E_{s} *$ is a normalized shelf energy that depends on sulfur content. Eliminating ${ }_{v} E_{s} *$ from (1) and (2), one obtains (3) which is equivalent to relation (ii) above.

$$
f \equiv{ }_{v} E_{s L} /{ }_{v} E_{s T}=\left(R_{L} / R_{T}\right)\left(R_{T}+\alpha\right) /\left(R_{L}+\alpha^{\prime}\right)
$$

This equation may be rewritten using $R=R_{L} \cdot R_{T}$,

$$
f=[\sqrt{ } R+\alpha \sqrt{ } Q] /\left[\sqrt{ } R+\left(\alpha^{\prime} / \sqrt{ } Q\right)\right]
$$

where $Q$ is the cross rolling ratio, $R_{L} / R_{T}$. Equation (4) may be fitted to measured $f^{\prime}{ }_{\mathrm{s}}$ (for example, Fig. 9 of Ref. 5)) taking $\alpha$ and $\alpha^{\prime}$ as adjustable parameters. Figure 1 is an example of such fitting, which shows satisfactory agreement for industrial $Q$ range. The fact that $\alpha$ is larger than $\alpha^{\prime}$ in Fig. 1 suggests that sulfide is more elongated in $L$ direction than in $T$ direction under same rolling ratio because of the generally lower rolling temperature in the final rolling. ${ }^{6,7)}$

The relationships so far obtained make possible the calculation of ${ }_{v} E_{s}{ }^{*}$ which is independent of $R$ and $Q$. Although a quick glance at mill data suggests ${ }_{{ }_{v}} E_{s L}+$ $\left.{ }_{v} E_{s T}\right) / 2$ or $\sqrt{ }_{v} E_{s L} \cdot{ }_{v} E_{s T}$ to be constant (Fig. 10 of Ref. $5)$, the following relation is obtained for the former assuming $\alpha=\alpha^{\prime}$.

$$
\frac{{ }_{v} E_{s L}+{ }_{v} E_{s T}}{2}=\left[1+\frac{\alpha}{2 \sqrt{ } R \cdot Q}(Q+1)\right]{ }_{v} E_{s}^{*} \cdot \cdots
$$

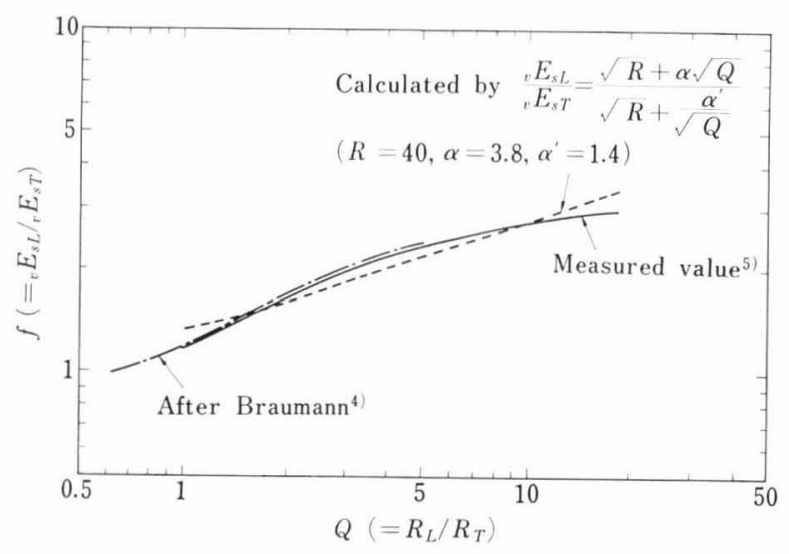

Fig. 1. Comparison of the measured and calculated ratio of shelf energy longitudinal to transverse

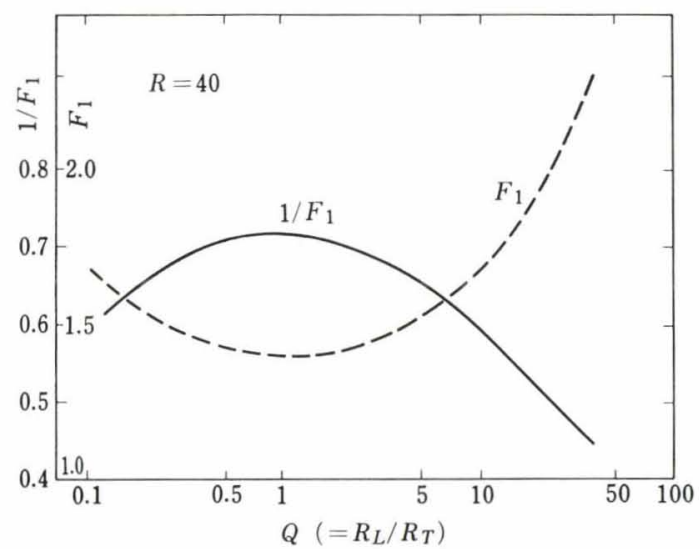

Fig. 2. Example of calculated correction factor $\left(F_{1}\right)$ of shelf energy

$$
R=40, \alpha=\alpha^{\prime}=2.5
$$

* $\quad$ Received May 31, 1972.

** Fukuyama Works, Nippon Kokan K. K., Kokan-cho, Fukuyama 720.

*** Technical Research Center, Nippon Kokan K. K., Minamiwatarida-cho, Kawasaki-ku, Kawasaki 210. 
Equation (5) indicates that the arithmetic average of ${ }_{v} E_{s L}$ and ${ }_{v} E_{s T}$ is influenced by $R$ and $Q$. Figure 2 is a plot of [ ] in Eq. (5) (denoted as $F_{1}$ ) and $1 / F_{1}$ for the case when $R=40$ and $\alpha=2.5$. $^{*}$ From Fig. 2 it can be seen that the arithmetic average becomes lower at $Q \simeq 1$ than at $Q \gg 1$, which agrees well with the observation in mill rolling. ${ }^{5)}$

It is also possible to estimate ${ }_{v} E_{s T}\left(\right.$ or ${ }_{v} E_{s L}$ ) for an arbitrary set of sulfur content and $Q$ from Eq. (6) under the condition of a constant total rolling ratio if $\mathrm{S} \%{ }_{v} E_{s}{ }^{*}$ (Fig. 11 of Ref. 5)) and Q-f (Fig. 9 of Ref. 5)) relations are established from mill data.

$$
{ }_{v} E_{s T}=\frac{2}{f+1} \cdot F_{1} \cdot{ }_{v} E_{s}^{*}
$$

Similar relations may be obtained for the geometrical average though they are not elaborated here.

\section{REFERENCES}

1) H. A. Vogels and F. Brüning: Arch. Eisenhüttenw., 35 (1964), 115

2) W. Dahl, H. Hengstenberg, and C. Düren: Stahl u. Eisen, 88 (1968), 364

3) J. Degenkolbe and W. Neuhaus: Stahl u. Eisen, 83 (1963), 1294.

4) F. Braumann: Stahl u. Eisen, 83 (1963), 1356.

5) H. Matsubara, T. Osuka, I. Kozasu, and K. Tsukada: Trans. ISIJ, 12 (1972), 435.

6) I. Kozasu and H. Kubota: Trans. ISIJ, 11 (1971), 321.

7) W. Dahl, H. Hengstenberg, and C. Düren: Stahl u. Eisen, 86 (1966), 782. 